\title{
An Identification of the Knowledge Structure on the Resilience of Caregivers of People with Dementia using a Text Network Analysis
}

\author{
Kim, Eun Young ${ }^{1} \cdot$ Chang, Sung $\mathrm{Ok}^{2}$ \\ ${ }^{1}$ Lecturer, Red Cross College of Nursing, Chung-Ang University, Seoul \\ ${ }^{2}$ Professor, College of Nursing \& BK21 FOUR R\&E Center for Learning Health Systems, Korea University, Seoul, Korea
}

\begin{abstract}
Purpose: The purpose of this study is to identify the knowledge structure of the concept of resilience of dementia caregivers and to understand core topics and the trends in dementia caregiver resilience research over time. Methods: This study is a quantitative content analysis using text network analysis on the studies on resilience of caregivers of people with dementia. We searched the available literature in two scientific databases, PubMed and the Web of Science. Term frequency, the number of co-occurrence, and centrality were analyzed, and Latent Dirichlet Allocation (LDA) analysis was conducted with Net Miner program. Results: A total 187 articles were identified. The core keywords found were "care", "family", "support", "burden", "intervention", "health", "stress", "relationship", "symptom", "depression", "partner", and "quality of life". Four main topics were identified through the LDA analysis, such as "support from family and social resources", "hardships to overcome", "building strength to overcome adversity", and "coping with negative emotions". There was only one study before 2000 , but the number of studies steadily increased to 50 for the years 2011 2015, and 111 for the years 2016 2020. Conclusion: This is the first study to analyze the knowledge structure and research trend of resilience research of caregivers of people with dementia through social network analysis and topic modeling. This study provides a scientific basis of the future studies in Korea. Moreover, it is meaningful in that it brought a comprehensive understanding of the studies by comprehensively analyzing the studies of the resilience of caregivers of people with dementia.
\end{abstract}

Key Words: Social networking; Resilience/psychological; Dementia; Caregivers; Humans

\section{INTRODUCTION}

The prevalence of dementia is steadily increasing with the growth of the world's aged and aging populations, creating a serious global problem [1]. Age is one of the most important risk factors for dementia, and the majority of people with dementia are composed of those aged 65 or older [2]. The prevalence of dementia among adults over the age of 65 in Korea is estimated to increase rapidly from $10.2 \%$ in 2018 to $16.1 \%$ in 2050 [2]. With this trend, the burden on formal and informal caregivers is expected to increase steadily [3]. According to the Lee's study [4], medical expenses in Korea are steadily increasing overall, and the disease that accounts for the largest proportion of them is dementia. In the post COVID-19 era, the number of people with dementia is expected to increase more rapidly due to social disconnection of people with dementia [5,6], and the responsibility of caregivers and social costs are also expected to increase.

Due to various physical and behavioral problems shown in people with dementia, caregivers face challenges, such as chronic stress and social isolation [7]. In addition, caregivers have various aspects of the burden, including physical, psychological, financial burdens as well as 'sandwich creation issues' (i.e. the need for taking care of both children and parents) [7]. Although caregivers of people with dementia face many difficulties, some caregivers appear to experience fewer negative consequences from caregiving than others [8] or experience high levels of satisfaction in their caregiving role [9] and

Corresponding author: Chang, Sung Ok https://orcid.org/0000-0003-2710-4291

College of Nursing, Korea University, 145 Anam-ro, Seongbuk-gu, Seoul 02841, Korea.

Tel: +82-2-3290-4918, Fax: +82-2-928-9108, E-mail: sungok@korea.ac.kr

Received: Dec 15, 2020 / Revised: Jan 26, 2021 / Accepted: Feb 14, 2021

This is an open access article distributed under the terms of the Creative Commons Attribution Non-Commercial License (http://creativecommons.org/licenses/ by-nc/3.0), which permits unrestricted non-commercial use, distribution, and reproduction in any medium, provided the original work is properly cited. 
positive health outcomes [10]. This has to do with the concept of "resilience", and many studies are increasingly focused on caregivers who are able to recover, resist or adapt to the needs of physical and psychological care [11]. Improving the resilience of caregivers can reduce the caregiving burden [12] and improve the psychological health of people with dementia [13]. Despite the increasing number of research on resilience of caregivers of people with dementia, no systematic attempt has been made to comprehensively analyze the research on resilience of caregivers of people with dementia. In order to provide deeper insights into the current dementia caregiver research, there is a need to examine the knowledge structure of the existing research and understand research trends and relationships among the core research concepts related to resilience in dementia caregivers [14].

Text network analysis corresponds to quantitative content analysis and is useful for discovering key topics based on the relationships between word co-occurrences in large-scale texts, interpreting the meaning of potential contexts, and visualizing their knowledge structures [15]. The knowledge structure is a contextual structure comprising research concepts that can show the boundaries or development process of knowledge [16]. Major research concepts discovered through text network analysis are regarded as the theoretical basis of academic fields that include the core topics of knowledge structures [16]. In addition, analyzing the trend of research that changes over time enables systematic understanding and serves as a compass that suggests future research directions [17].

This study applied the text network analysis to the identified set of abstracts of previous studies on the resil- ience of caregivers of people with dementia to understand the knowledge structure of the concept of resilience of caregivers of people with dementia. We also aimed to analyze the text network and topic modeling to understand the core topics and main meanings and explore the research trends according to the structure and time. Through this method, we anticipated that we could provide a comprehensive view of the resilience of caregivers of people with dementia and identify the research areas necessary to improve the quality of life among caregivers of people with dementia. Moreover, we will be able to suggest future directions for nursing research on resilience of caregivers of people with dementia.

\section{METHODS}

\section{Study Design}

This study is a quantitative content analysis using text network analysis on the English abstract of the identified studies on resilience of caregivers of people with dementia.

\section{Study Process}

Figure 1 shows the study flow. The Net Miner version 4.3 (Cyram, Seongman, South Korea) program was used for data analysis. The detailed research process is described below.

\section{Data Collection}

Data collection in this study refers to the collection of

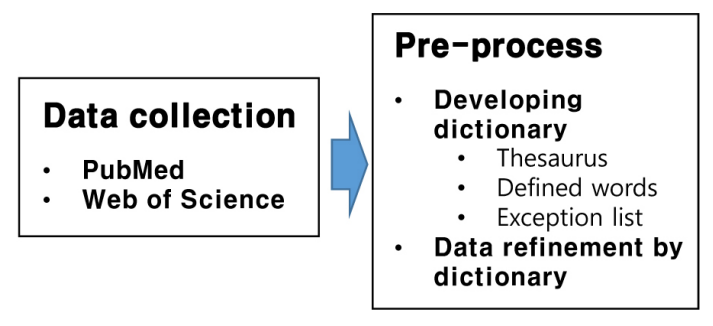

LDA=Latent Dirichlet Allocation.

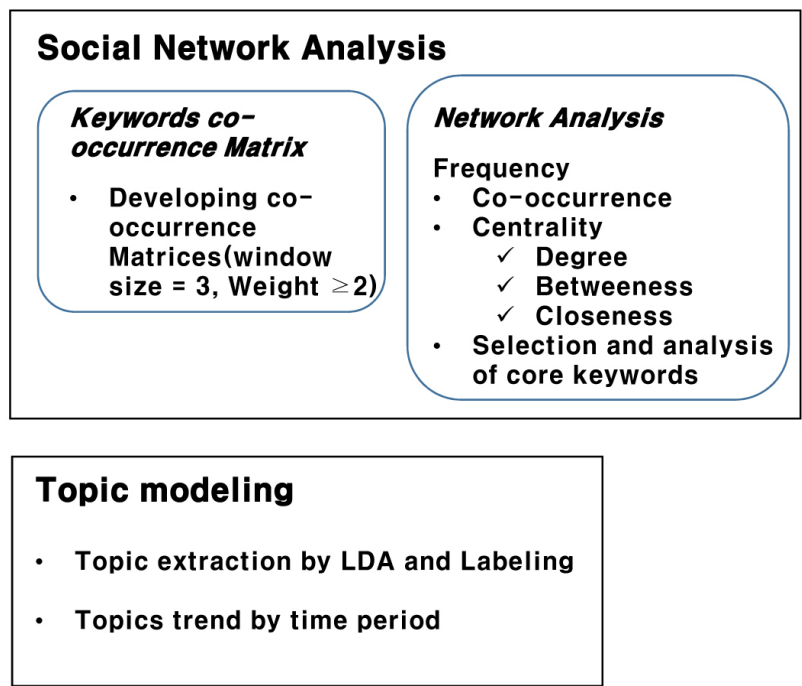

Figure 1. The overall research process. 
abstracts of the studies identified through a systematic search of scientific databases. The data collection was conducted from May 25th to June 5th, 2020. The inclusion criteria were studies that were: (a) including "dementia", "caregiver", and "resilience" in the title, abstract, and key words, (b) published in peer-reviewed journals, and (c) published in English. Since there have been no similar studies so far, we did not limit our search based on publication years for broad trend research. The exclusion criteria were: (a) articles without abstracts, and (b) not based in human subjects.

To identify relevant articles, we conducted a systematic search of PubMed and the Web of Science. A total of 269 articles were identified. After removing duplicates and articles without abstracts, the abstracts of 187 articles were included for the final analysis (Figure 2).

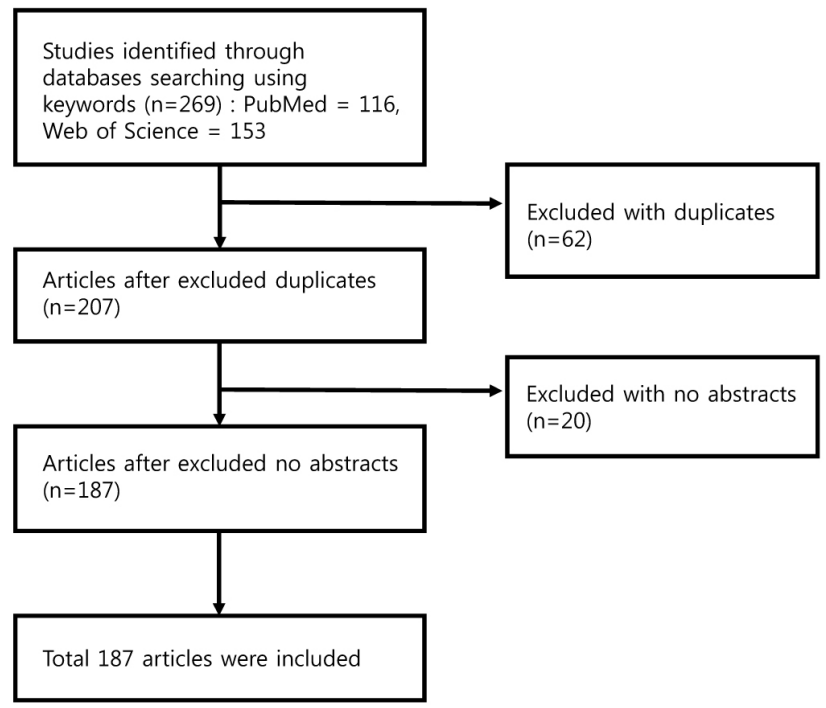

Figure 2. The data collection process.

\section{Pre-processing}

For a text network analysis, accurate keyword extraction is paramount [18]. Two researchers, KEY and CSO, registered exceptional words dictionary, defined words dictionary, and thesaurus words dictionary to Net Miner version 4.3 program. Words that are not related to the research topic and may confuse their meaning are registered on an exceptional words dictionary, while some word phrases are registered in a defined words dictionary so that their meaning is communicated clearly [18]. Words with similar meanings have been registered in the thesaurus words dictionary [18]. This work was performed independently by the aforementioned two researchers, after which we compared each other's work and revised it after discussing the differences.

\section{Network Analysis}

The main words were extracted through text network analysis. In this study, the criterion for the simultaneous appearance of keyword pairs was set to "window size=3", which means when two keywords appear side by side in one sentence or appear with another keyword in between them [17]. A network analysis was performed using the 1-mode matrix of each text of the generated literature abstract. Term frequency, the number of co-occurrence, and centrality were analyzed. Term frequency can confirm the overall flow of the study [19]. Term frequency analysis is performed through counting the total number of occurrences of terms, and it is possible to identify the central words that are important in each document [19]. Co-occurrence refers to the number of two keywords that appear together within a document [18]. Centrality is a relative indicator of the degree to which keywords are centered in the network [19]. Degree centrality, betweenness centrality, and closeness centrality were used to figure out the important nodes within the network [18]. Degree centrality measures the number of links each node in the language network has with other nodes, and it means that keywords with high degree centrality occupy a central position to connect other keywords [18]. Between centrality is a value that measures the degree to which one keyword plays a mediating role between other keywords, and the higher the value, the higher the control over information exchange over the language network $[18,19]$. Closeness centrality measures centrality based on the distance between keywords and refers to the distance between not only directly connected nodes but all indirectly connected keywords [18]. The higher the closeness centrality value, the closer it is to other keywords [18].

\section{Topic Modeling}

The LDA (Latent Dirichlet Allocation) analysis was applied to identify potential major topics. It is a method that extracts topics based on words with high relevance from a large amount of data. The association between words is calculated based on the frequency of co-occurrences in the document set [20]. By figuring out the percentage of topics per document and the probability that words will be included in the topic, you can estimate the topics hidden in the document [21]. Two researchers (KEY, CSO) 
independently analyzed the keywords assigned to the topic and named each topic by re-reading the original text related to the keyword, then comparing the results together and discussing them to determine the name of each topic. The name of each topic was reviewed by five nurses specialized in gerontology research to determine whether the relevance of the topic according to the key word and naming of the topic is appropriate. Then, the name of the topic was confirmed through the final discussion between the two researchers (KEY, CSO). Finally, by dividing the studies every 5 years, we calculated the number of research in each topic area and analyzed the trends of hanges in the study topics over time according to the 5 -year periods.

\section{RESULTS}

We identified 187 studies related to the resilience of caregivers of people with dementia published from 1999 to 2020 .

\section{Keyword Analysis Related to the Resilience of Caregivers of People with Dementia}

From the identified studies, 550 keywords were extracted. From the pool of the identified keywords, the co-occurrence, term frequency, degree centrality, betweenness centrality, and closeness centrality of all keywords were analyzed, and the top 50 keywords were extracted in each item. of the 50 keywords extracted in each item, 24 keywords were included in all of these keyword analysis items, which were treated as the core keywords of this study. Among the three centralities, the keywords that appeared most commonly across studies were "care", "family", "support", "burden", "intervention", "health", "stress", "relationship", "symptom", "depression", "partner", "role" and "quality of life"(Table 1). Looking at the degree centrality presented in Table 1, care (68) has the highest degree centrality, followed by family (39), burden (37), support (36), and stress (35). Keywords with high degree centrality indicate a central position to connect other keywords [17].

In terms of betweenness centrality, care (0.29) was the highest, followed by family (0.20), support (0.19), stress (0.19), burden (0.18), and relationship (0.18). It means that keywords with high betweenness centrality played a role in mediating other keywords [17]. The keyword with the highest closeness centrality was family (0.37), followed by intervention (0.35), stress $(0.35)$, burden $(0.34)$, care $(0.33)$, and health (0.33). The higher closeness centrality means that the distance from other keyword is closer.[17]. The keywords included in the top in all three centralities were "care", "family", "support", "burden", "intervention", "health", "stress", "relationship", and "depression". "Symptoms" were high only in closeness centrality and 'partner' only in betweeness centrality.

\section{Topics Related to the Resilience of Caregivers of People with Dementia}

\section{1) Labeling of topics related to the resilience of caregivers of people with dementia}

By performing topic modeling, four major topics were identified, and keywords assigned to these topics were checked (Figure 3). The topics identified were 1) support from family and social resources, 2) hardships to overcome, 3) building strength to overcome adversity, and 4) coping with negative emotions. Topics 1,3 and 4 had links with the keyword "care"; topics 3 and 4 had links with three keywords, "care", "health", and "relationship"; topics 2 and 3 had a link with the keyword "partner"; and topics 2 and 4 were connected to the keyword "stress".

\section{2) Analysis of trends in research on the resilience of de- mentia caregivers}

In order to examine the research trends, the identified articles were divided into 5-year periods, and the distribution of topics derived through topic modeling was analyzed. The analysis shows that the total number of studies gradually increased to $1,3,17,50$, and 116 cases in each 5-year period from 1999 to 2020. Looking at the trends of each topic according to the period, it was observed that the number of papers related to all four topics increased over time. We can see that topic 1 increased with the steepest slope, and topic 2 increased at the lowest rate. The slope of the graph has increased since 2006, indicating that active research has been conducted recently. Until 2005, only four studies had been conducted, so it was not possible to compare the proportions, so these four studies were excluded from the comparison of the percentage of each topic. Between 2006 and 2010, the proportion of published articles for topic1, 2, 3, and 4 was $41.2 \%, 11.8 \%, 5.8 \%$, and $41.2 \%$, respectively. Between 2016 and 2020, topic $1,2,3$, and 4 was $37.8 \%, 14.4 \%$, $21.6 \%$, and $26.1 \%$ (Figure 4 ).

\section{DISCUSSION}

This study identified the key research topics and analyzed keywords and research trends by performing text 
Table 1. Keywords Analysis

\begin{tabular}{|c|c|c|c|c|c|}
\hline Keywords & Co-occurrence & Term frequency & $\begin{array}{l}\text { Degree of } \\
\text { centrality }\end{array}$ & $\begin{array}{c}\text { Betweenness } \\
\text { centrality }\end{array}$ & $\begin{array}{l}\text { Closeness } \\
\text { centrality }\end{array}$ \\
\hline Care & 138 & 282 & 68 & 0.29 & 0.33 \\
\hline Family & 95 & 199 & 39 & 0.20 & 0.37 \\
\hline Support & 84 & 187 & 36 & 0.19 & 0.32 \\
\hline Burden & 72 & 155 & 37 & 0.18 & 0.34 \\
\hline Intervention & 63 & 132 & 23 & 0.16 & 0.35 \\
\hline Health & 58 & 129 & 27 & 0.15 & 0.33 \\
\hline Stress & 52 & 118 & 35 & 0.19 & 0.35 \\
\hline Relationship & 45 & 97 & 29 & 0.18 & 0.32 \\
\hline Symptom & 39 & 92 & 17 & 0.10 & 0.32 \\
\hline Depression & 38 & 73 & 22 & 0.17 & 0.30 \\
\hline Partner & 28 & 70 & 16 & 0.12 & 0.25 \\
\hline Role & 25 & 66 & 9 & 0.08 & 0.29 \\
\hline Quality of life & 24 & 63 & 13 & 0.03 & 0.24 \\
\hline Alzheimer & 20 & 57 & 10 & 0.06 & 0.25 \\
\hline Resource & 19 & 56 & 15 & 0.03 & 0.25 \\
\hline Need & 20 & 54 & 11 & 0.09 & 0.23 \\
\hline Service & 18 & 50 & 12 & 0.04 & 0.28 \\
\hline Grief & 15 & 37 & 12 & 0.05 & 0.27 \\
\hline Individual & 12 & 37 & 14 & 0.09 & 0.21 \\
\hline Community & 13 & 32 & 9 & 0.02 & 0.23 \\
\hline System & 9 & 24 & 8 & 0.01 & 0.25 \\
\hline Religion & 7 & 17 & 11 & 0.03 & 0.26 \\
\hline End of life & 4 & 12 & 8 & 0.02 & 0.28 \\
\hline Conflict & 3 & 9 & 7 & 0.02 & 0.23 \\
\hline
\end{tabular}

network analysis and topic modeling based on the identified 187 articles on the resilience of dementia caregivers, from 1999 to 2020. A knowledge structure is a contextual structure between research concepts [16]. Visualizing the knowledge structure helps understanding the subject, shows the development process, and is regarded as the theoretical basis of the academic field [22]. The results of this study can provide a scientific basis because a vast amount of scientific research papers were collected as text data and searched through quantitative content analysis methods.

\section{Knowledge Structure by Extracting Key Keywords from the Research on the Resilience of Caregivers of People with Dementia}

The keyword analysis was chosen in this study as a method to grasp the properties of semantic relationships in the research about the resilience of caregivers of people with dementia. In the keyword analysis, it can be seen that the term frequency and co-occurrence of "family", "support", "burden", "relationship", "partner", and "role" are high. This can be interpreted as caregivers of people with dementia have a great significance in the formation of relationships and division of roles, such as family, support, relationship, and partner. This is similar to the results of a previous resilience study that showed caregivers acquired the power to overcome situations through meaningful relationships [23]. Compared with the network analysis study of dementia conducted in 2017 [24], co-occurrence was high in the keywords representing the patient's condition, such as cognition, memory, depression, the elderly, Parkinson, and cognitive impairment. In comparison with this, we found that the occurrences of the key- 


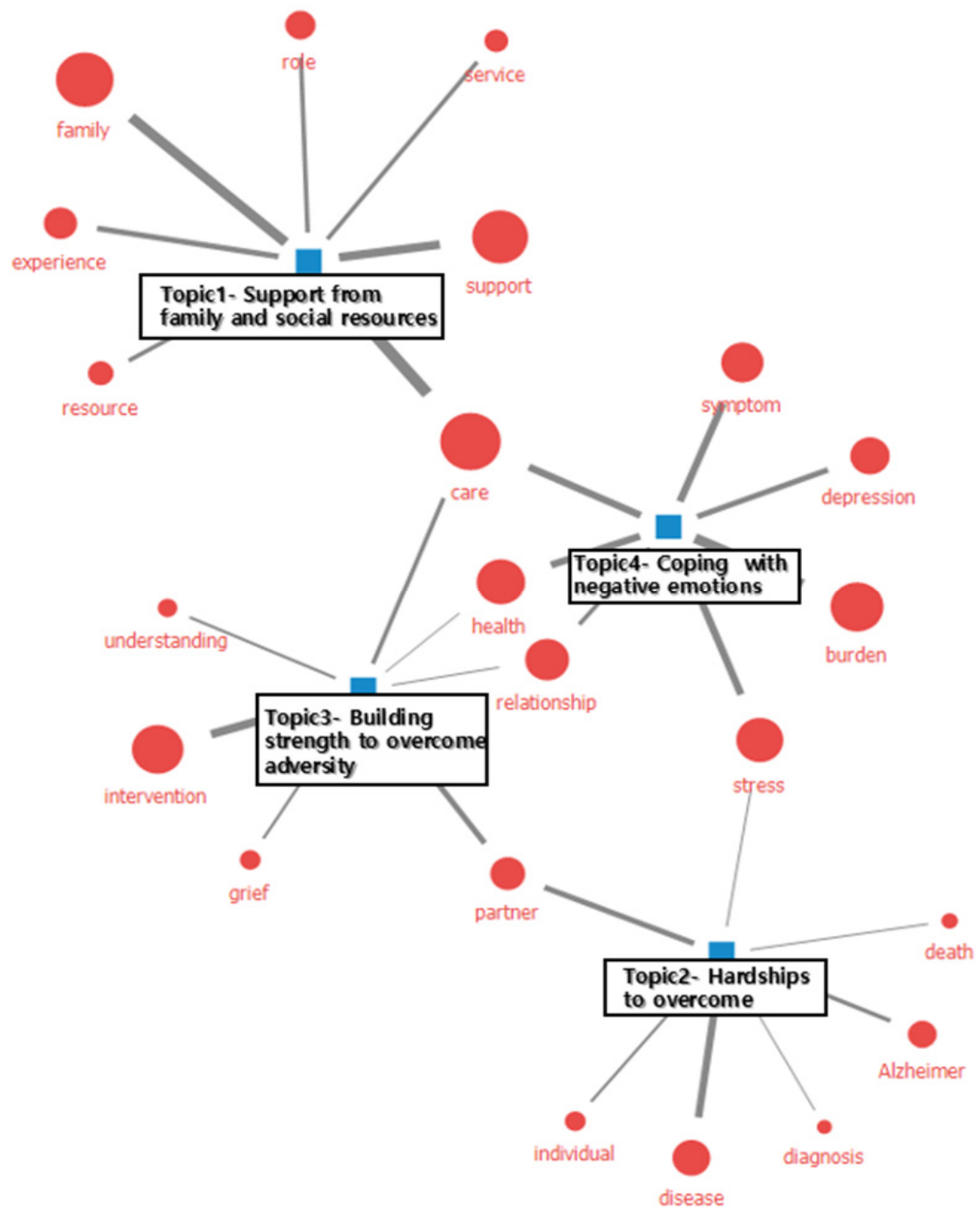

Figure 3. Topics related to the resilience of caregivers of people with dementia.

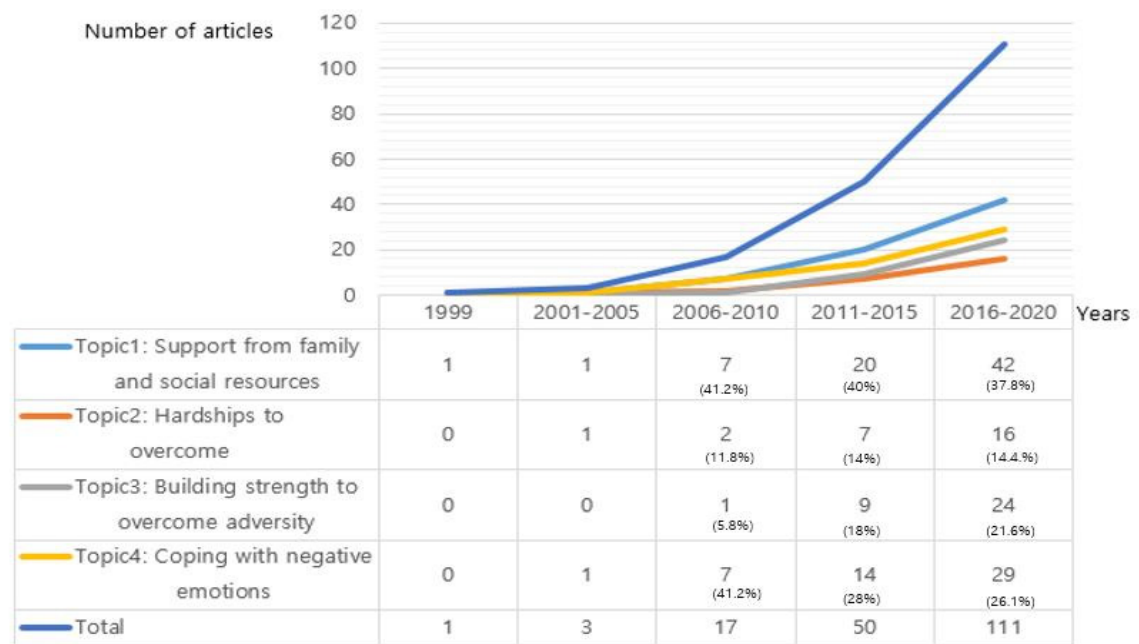

Figure 4. Trends in research on the resilience of caregivers of people with dementia by periods, from 1999 to 2020. 
words related to human relations with patients, support systems, roles, and meaning of life were high in our study. This can be seen as a characteristic of research related to the resilience of caregivers of people with dementia.

\section{Research Trend by Topic Modeling of Research Related to the Resilience of Caregivers of People with Dementia}

Through topic analysis by time period, the overall trend of research was examined. We have identified four topics: 1) support from family and social resources, 2) hardships to overcome, 3) building strength to overcome adversity, and 4) coping with negative emotions. Also, we found that the number of studies has been increasing in the field of dementia caregiver resilience over time. This reflects the trend of the rapidly increasing number of people with dementia that has led to an increased number of caregivers dealing with everyday challenges related to dementia caregiving.

\section{1) Support from family and social resources}

Care, family, support, role and resource appeared as related keywords for the topic, "support from family and social resources". In several studies of resilience to date, family, important human relationship and supportive resources have been found to have a positive effect on overcoming a crisis and have been identified as one of the most important attributes of resilience $[25,26]$. In this study, the result that this topic has been studied at the highest rate and that research is steadily increasing shows that many experts recognize this topic as a very important one in caregiver resilience. According to Ong's study, social support served as a full mediator between resilience and caregiver burden, it was recommended for healthcare providers to promote supportive social network including family and friends that may improve caregiver resilience and reduce caregiver burden [12]. Thus, it is necessary to discuss ways to develop policies that support the importance of family and social resources by providing caregivers with practical help in relieving the caregiving burden.

\section{2) Hardships to overcome}

In the topic, "hardships to overcome," stress, disease, partner, Alzheimer, and death were identified as the core keywords. These keywords represent negative feelings among caregivers and were named as "hardships to overcome" because they were judged to be related to the factors to overcome. In previous studies, caregivers reported that they experience high levels of stress; among several stressors, the illness and death of people with dementia were considered strong stressors for caregivers [27], which supports this study's results. As time goes on, research pertaining to the topic 2 has been also steadily increasing, but the rate of increase is the lowest compared to the rates for other topics. In order to enhance the resilience of caregivers, one of the most important points is that identifying the challenges they face first. Since modern society is rapidly changing and new stressors can occur, research needs to be conducted to uncover unknown stressors, and this could help clarify the challenges faced by caregivers. In recent years, because of the coronavirus pandemic, people with dementia and caregivers are facing another difficult situation than before, such as social disconnection $[5,6]$, and this may vary from country to country. Therefore, in the future, it is necessary to conduct research that can identify factors causing difficulties and reflecting a unique Korean situation and that seeks various ways to overcome those challenges.

\section{3) Building strength to overcome adversity}

In the topic, "building strength to overcome adversity," care, intervention, health, relationship, partner, understanding, and grief were identified as the core keywords. This topic was interpreted as an effort to overcome adversity. It focuses on the need for developing one's strength for overcoming crises in a variety of ways, such as overcoming through meaningful human relationships, trying to understand difficulties and sadness, and overcoming through mediation, rather than just effort. In the case of the topic 3, no research was conducted until 2008, and it has been steadily increasing from 2009 to the latest. Intervention studies, such as adaptive coping, stress management, and other interventions that highlight strengths and resilience, have been conducted. Although not included in the results of this study, to compare them with domestic trends, we searched and compared related studies in domestic journals. The result was, most studies on the resilience of caregivers of people with dementia in Korea were included in the topic 3, and most of the research subjects were family members [28,29]. Therefore, it is necessary to expand the scope of research to more diverse subjects and topics that can be of practical help to Korean caregivers of people with dementia.

\section{4) Coping with negative emotions}

Care, burden, health, relationship, stress, symptom, and depression were identified as the core keywords for the topic 4, "coping with negative emotions". The core 
keywords that emerged from this topic is related to coping with various negative emotions, such as burden, stress, and depression that a caregiver feels while caring for a patient and was thus named "coping with negative emotions". In this topic, it was revealed that it is important not only to understand those negative emotions, but to deal effectively with them. There was no research until 2001; however, research on this topic started increasing at a relatively rapid rate compared to other topics. Despite this trend, not many studies on the resilience of caregivers of people with dementia have been conducted in Korea. Because improving caregiver resilience can have a positive effect on psychological health [12,13], more related research should be performed in Korea in the future.

Based on the above results, we would like to suggest the direction of the domestic research about resilience of caregivers of people with dementia. First, it needs to be expanded to a variety of research subjects, including for$\mathrm{mal}$ and informal guardians. Second, it is necessary to understand the difficulties that caregivers of domestic people with dementia experience and the socio-cultural factors in Korea that affect dementia patient caregivers. Third, research is needed to find ways to overcome difficulties for caregivers, and a discussion on how to receive social support will be required. This can ultimately help improve the quality of life of the caregivers of people with dementia, and this can be expected to improve the health of people with dementia.

As a limitation of this study, the literature search was based only through PubMed and Web of Science to find the global research trends. We did not search potential studies through Korean databases which resulted in the exclusion of domestic studies. This limited our ability to identify research trends in Korea and compare international research trends with domestic research trends. Future research should focus on diversifying the literature search engine to allow more in-depth discussions by comparing domestic and overseas.

\section{CONCLUSION}

As the number of people with dementia is increasing, the role of caregivers is gradually increasing, and the burdens and difficulties of caregivers are also increasing. Research on caregiver's resilience is an important concept that can ultimately promote the health of people with dementia as well as the quality of life of the caregiver. This study is the first to analyze the knowledge structure and trends of resilience research of caregivers of people with dementia using social network analysis and topic model- ing. This study tries to provide a scientific basis for future research in Korea by providing an understanding of international trends. In addition, it is significant in that it brings a comprehensive understanding of the study by comprehensively analyzing the resilience studies of caregivers of people with dementia.

\section{CONFLICTS OF INTEREST}

The authors declared no conflict of interest.

\section{AUTHORSHIP}

Study conception and design acquisition - KEY and CSO; Data collection - KEY and CSO; Analysis and interpretation of the data - KEY and CSO; Drafting and critical revision of the manuscript KEY and CSO; Final Approval - CSO.

\section{ACKNOWLEDGEMENT}

This research was supported by a National Research Foundation of Korea grant funded by the Korea government(Ministry of Science and ICT) (No. NRF-2019R1A2C1086122).

\section{REFERENCES}

1. Weller J, Budson A. Current understanding of Alzheimer's disease diagnosis and treatment. F1000Research. 2018;7(1161):1-9. https://doi.org/10.12688/f1000research.14506.1

2. National Institute Dementia. The present condition of dementia [Internet]. Seoul: National Institute Dementia; 2020 [updated 2020; cited 2020 Oct 20]. Available from: https://www.nid.or.kr/info/diction_list2.aspx?gubun=0201

3. Alzheimer's Association. Alzheimer's and dementia facts and figures [Internet]. Chicago: Alzheimer's Association; 2020 [updated 2020; cited 2020 Oct 15]. Available from: https://www.alz.org/alzheimers-dementia/facts-figures

4. Lee YJ. The effect of facility characteristics of geriatric hospitals on long stay dementia patients. [master's thesis], [Seoul]: Yonsei University; 2018. 15 p.

5. Suh GH. What explains COVID-19's Lethality for the elderly?: community care strategies for the vulnerable elderly. Journal of Health Technology Assessment. 2020;8(1):9-13.

https://doi.org/10.34161/johta.2020.8.1.002

6. Bynn TS. "Concerns about the explosion of dementia patients with disconnected social activities in the post-corona era...". [Internet]. Seoul:The Korea Times;2020 [updated 2020 Sep 4; cited 2021 Jan 8]. Available from:

https://www.hankookilbo.com/News/Read/A20200904153 20002449?did=NA

7. Cuyler R. Caregiver Stress in Dementia [Internet]. Texas: Brain Check; 2019 [updated 2019 May 15; cited 2020 Oct 20]. Avail- 
able from:

https://braincheck.com/articles/caregiver-stress-dementia/

8. O'Rourke N, Kupferschmidt AL, Claxton A, Smith JZ, Chappell

N, Beattie BL. Psychological resilience predicts depressive symptoms among spouses of persons with Alzheimer disease over time. Aging \& Mental Health. 2010;14(8):984-93. https://doi.org/10.1080/13607863.2010.501063

9. Hellström I, Nolan M, Lundh U. Sustaining couplehood: spouses' strategies for living positively with dementia. Dementia. 2007;6(3):383-409.

https://doi.org/10.1177/1471301207081571

10. Wilks SE, Little KG, Gough HR, Spurlock WJ. Alzheimer's aggression: influences on caregiver coping and resilience. Journal of Gerontological Social Work. 2011;54(3):260-75. https://doi.org/10.1080/01634372.2010.544531

11. Herrman H, Stewart DE, Diaz-Granados N, Berger EL, Jackson B, Yuen T. What is resilience? The Canadian Journal of Psychiatry. 2011;56(5):258-65. https://doi.org/10.1177/070674371105600504

12. Ong HL, Vaingankar JA, Abdin E, Sambasivam R, Fauziana R, Tan M-E, et al. Resilience and burden in caregivers of older adults: moderating and mediating effects of perceived social support. BMC Psychiatry. 2018;18(1):27.1-9. https://doi.org/10.1186/s12888-018-1616-z

13. Yang F, Ran M, Luo W. Depression of persons with dementia and family caregiver burden: finding positives in caregiving as a moderator. Geriatric \& Gerontology International. 2019; 19(5):414-8. https://doi.org/10.1111/ggi.13632

14. Park CS, Park E. Identification of knowledge structure of pain management nursing research applying text network analysis. Journal of Korean Academy of Nursing. 2019;49(5):538-49. https://doi.org/10.4040/jkan.2019.49.5.538

15. Paranyushkin D. Identifying the pathways for meaning circulation using text network analysis. Venture Fiction Practices. 2011;2(4):1-21.

16. Kwon SY, Bae KR. A study on the knowledge structure of cancer survivors based on social network analysis. Journal of Korean Academy of Nursing. 2016;46(1):50-8. https://doi.org/10.4040/jkan.2016.46.1.50

17. Kim Y, Jang SN. Mapping the knowledge structure of frailty in journal articles by text network analysis. PLoS ONE. 2018;13 (4):e0196104. https://doi.org/10.1371/journal.pone.0196104
18. Lee SS. A content analysis of journal articles using the language network analysis methods. Journal of the Korean Society for Information Management. 2014;31(4):49-68.

https://doi.org/10.3743/KOSIM.2014.31.4.049

19. Kim YH, Kim YJ. Social network analysis. 4th ed. Seoul: Parkyoungsa; 2016. 91 p, 132 p.

20. Nam HD, Nam TW. An exploratory study of platform government in Korea: topic modeling and network analysis of public agency reports. Journal of Digital Convergence. 2020;18(2): 139-49. https://doi.org/10.14400/JDC.2020.18.2.139

21. Kim JJ. Literature review on humanitarian logistics using network analysis and topic modeling [master's thesis], [Busan]: Busan University; 2020. 29 p.

22. Lee SK, Jeong S, Kim HG, Yom YH. A social network analysis of research topics in Korean nursing science. Journal of Korean Academy of Nursing. 2011;41(5):623-32. https://doi.org/10.4040/jkan.2011.41.5.623

23. Kim EY, Lee YN, Chang SO. How do patients on hemodialysis perceive and overcome hemodialysis?: Concept development of the resilience of patients on hemodialysis. Nephrology Nursing Journal. 2019;46(5):521-30.

24. Han JH, Yom YH. Social network analysis on mapping the knowledge structure of dementia research. Journal of Korean Gerontological Nursing. 2017;19(2):69-80.

https://doi.org/10.17079/jkgn.2017.19.2.69

25. Windle G. What is resilience? a review and concept analysis. Reviews in Clinical Gerontology. 2011;21(2):152-69. https://doi.org/10.1017/S0959259810000420

26. Earvolino-Ramirez M. Resilience: a concept analysis. Nursing Forum. 2007;42(2):73-82. https://doi.org/10.1111/j.1744-6198.2007.00070.x

27. Chiao C, Wu H, Hsiao C. Caregiver burden for informal caregivers of patients with dementia: a systematic review. International Nursing Review. 2015;62(3):340-50. https://doi.org/10.1111/inr.12194

28. Bang M, Kim O. Effect of the Family Resilience Reinforcement Program for Family Caregivers of the Elderly with Dementia. Korean Journal of Adult Nursing. 2016:28(1):71-82. https://doi.org/10.7475/kjan.2016.28.1.71

29. Yeon BK, Yun SK, Yoon HJ. Evaluation of the effects of supportive program for family caregivers of the elderly with dementia. The Journal of Korean Long Term Care. 2019;7(2):4563. https://doi.org/10.32928/TJLTC.7.2.3 